\title{
Efeito do tipo de fixador e tempo de fixação na morfologia de folículos pré-antrais ovarianos bovinos
}

\section{Effect of fixative type and fixation time on the morphology of bovine preantral ovarian follicles}

\author{
Joabel Tonellotto dos Santos ${ }^{1}$; Katia Cristina Silva-Santos ${ }^{2}$; \\ Evelyn Rabelo Andrade 3 ; Livia Aires Lisboa ${ }^{4}$; Carine Letícia Schneider ${ }^{5}$; \\ Alethéia Ciquini' ${ }^{6}$, Rogerio Ferreira ${ }^{7}$; \\ Jandui Escarião da Nóbrega Junior ${ }^{8}$; Marcelo Marcondes Seneda ${ }^{9 *}$
}

\section{Resumo}

\begin{abstract}
Na rotina de avaliação histológica, os fixadores mais adequados para o tecido ovariano para que se obtenha uma adequada análise morfológica do ovário e de suas células ainda não foram determinados. O correto processamento do tecido é fundamental para evitar erros de interpretação, como confundir degeneração folicular devido ao processo fisiológico de atresia folicular com sinais de degeneração devido à má fixação do tecido. Este estudo teve como objetivo identificar qual o melhor fixador e melhor período de fixação para análise morfológica da unidade funcional do ovário: o folículo. Para isso foram utilizados ovários $(\mathrm{n}=5)$ de cinco vacas adultas. Cada ovário foi divido em 12 fragmentos e submetidos a seis diferentes fixadores em dois tempos (12 e 24h). Foram analisados 150 folículos ovarianos por tratamento. Os melhores resultados foram obtidos com os fixadores Carnoy e Bouin, que apresentaram maior $(\mathrm{P}<0,05)$ proporção de folículos viáveis após fixação por 12 ou $24 \mathrm{~h}(88$ e $83 \%$ para o fixador Carnoy vs. 79 e $73 \%$ para Bouin respectivamente). Conclui-se neste estudo que os fixadores Carnoy e Bouin são as melhores opções para preservar por até $24 \mathrm{~h}$ a morfologia de folículos pré-antrais em ovários bovinos.
\end{abstract}

Palavras-chave: Fixador, histologia, ovário, folículo pré-antral

\footnotetext{
Abstract

There are no efficient fixatives for histological analyses of ovarian tissue that permit an appropriate morphological analysis of ovary and their cells. The correct processing of the tissue is essential to avoid misinterpretation between follicular degeneration due to the physiological process of follicular atresia with signs of degeneration signals due to poor tissue fixation. This study aimed to identify the best fixative and better fixation period for morphological analysis of the functional unit of the ovary: follicle.

1 Doutorando na área de Reprodução Animal, UFSM. Santa Maria, RS. E-mail: joabelts@yahoo.com.br

2 Doutoranda na área de Reprodução Animal, Universidade Estadual de Londrina, UEL. Londrina, PR. E-mail: kati_cris@hotmail.

3 Bolsista DTI/CNPq, UEMS. Aquidauana, MS. E-mail: evelyn_andrade@yahoo.com

4 Pós-doutoranda na área de Reprodução Animal, UEL. Londrina, PR. E-mail: lisboavet@gmail.com

5 Discente de graduação em Medicina Veterinária, UEL. Londrina, PR. E-mail: carineschneider2@hotmail.com

6 Técnica de laboratório, UEL, Londrina, PR. E-mail: aac@uel.br

Prof. Dr. Adjunto da Universidade do Estado de Santa Catarina. UDESC, SC.E-mail: rferreira.sul@gmail.com

8 Dr. na área de Reprodução Animal, UFSM. Santa Maria, RS. E-mail: j.escariao@gmail.com

9 Prof. Dr. do Dept ${ }^{\circ}$ de Clínicas Veterinárias, Centro de Ciências Agrárias, UEL, Londrina, PR. E-mail: mseneda@uel.br Autor para correspondência
} com 
Ovaries $(\mathrm{n}=5)$ from five adult cows were collected from local abattoirs. Each ovary was divided into 12 fragments and submitted to six different fixatives in two different times (12 and 24h). It was analyzed 150 follicles per treatment. The best results were obtained with Carnoy' and Bouin's fixatives which had higher $(\mathrm{P}<0.05)$ proportion of viable follicles after fixation for 12 or $24 \mathrm{~h}(88$ and $83 \%$ for Carnoy's fixative vs. 79 e $73 \%$ for Bouin). It was concluded that Carnoy and Bouin fixatives are the best options to preserve for up to $24 \mathrm{~h}$ the morphology of preantral follicles in bovine ovaries.

Key words: Fixative, histology, ovary, preantral follicle

\section{Introdução}

O folículo é a unidade morfofuncional do ovário. É constituído por um oócito circundado por células da granulosa e da teca e possui atividade exócrina ou gametogênica. Os folículos pré-antrais representam mais de $90 \%$ de toda reserva de gametas femininos (SAUMANDE, 1991; FIGUEIREDO et al., 2007) e os oócitos inclusos nestes folículos representam excelente alternativa para a preservação e posterior propagação de animais de alto valor econômico ou em vias de extinção (CHAVES et al., 2008).

A análise histológica permite uma avaliação mais detalhada das estruturas foliculares. A base para uma boa preparação histológica é a fixação, que deve ser completa e adequada. Os principais objetivos da fixação incluem a inibição da autólise tecidual, coagulação ou endurecimento do tecido, preservação dos vários componentes celulares e tissulares, melhora na diferenciação óptica dos tecidos e, finalmente, facilitação da subsequente coloração (JUNQUEIRA; CARNEIRO, 2008). O tempo de fixação varia de acordo com o tamanho e constituição do tecido, poder de fixação e temperatura do fixador (CULLING; ALLISSON; BARR, 1985).

Diversos fixadores têm sido empregados, como acetona, metanol, Bouin, formalina a $10 \%$, periodatolisina, paraformoldeído, acetona-metil benzoatoxileno e EDTA (BAUMGARTNER et al., 1988). Quando não fixadas corretamente as células e seus constituintes apresentam-se distorcidas e retraídas, o que dificulta a avaliação histológica (FOX et al., 1985). A formalina a $10 \%$ para microscopia óptica e o aldeído glutárico em solução de 2 a $6 \%$ para microscopia eletrônica são os fixadores simples mais comumente utilizados (FOX et al., 1985). Os fixadores de natureza alcoólica necessitam menor tempo para fixação, o que acelera o período requerido para o processamento histológico, embora possam promover encolhimento, colapso e endurecimento dos tecidos pela coagulação das proteínas e ácidos nucléicos. O metanol ou ácido acético, classificados como fixadores desnaturantes ou coagulantes de proteínas condicionam aspecto diferente aos tecidos fixados, o que pode interferir negativamente nas análises estruturais (BUESA, 2008). O etanol 70\%, pouco oneroso e de fácil preparo, pode ser utilizado com sucesso para fixação de tecido glandular, além de órgãos como rim e próstata, permitindo a satisfatória inclusão em parafina (GILLESPIE et al., 2002).

Nos protocolos de histologia para tecido ovariano foi relatado o uso, com bons resultados, fixadores como o paraformaldeído a 4\% (SILVA et al., 2004), Bouin (ANDRADE et al., 2005) e Carnoy (MATOS et al., 2004). Entretanto, um consenso sobre o melhor fixador e tempo ideal para fixação e adequada preservação das estruturas ovarianas permanecem incertos. Visando facilitar estudos morfológicos do tecido ovariano, considerando a importância deste órgão para a reprodução, o objetivo deste estudo foi comparar o efeito de diferentes fixadores e tempos de fixação sobre a morfologia de folículos pré-antrais em fragmentos ovarianos bovinos processados para histologia.

\section{Material e Métodos}

\section{Coleta dos ovários}

Ovários $(n=5)$ de cinco vacas não gestantes adultas Bos taurus indicus (Nelore) foram coletados em abatedouros. Imediatamente após a coleta, os ovários foram lavados em solução salina $0,9 \%$ e 
divididos em 12 fragmentos com aproximadamente $9 \mathrm{~mm}^{3}(3 \times 3 \times 1 \mathrm{~cm})$ foram retirados do córtex de cada ovário. Em seguida, os fragmentos ovarianos foram imersos em seis diferentes fixadores por 12 ou 24 $\mathrm{h}$ (Figura 1). Os fragmentos foram acondicionados individualmente em frascos de vidro contendo aproximadamente 20 vezes o volume da solução fixadora. Os fixadores utilizados foram: Álcool 70\%
(A-12 e A-24 - GILLESPIE et al., 2002), Bouin (B12 e B-24 - ANDRADE et al., 2005), Carnoy (C-12 e C-24 - MATOS et al., 2004), Karnovsky (K-12 e K-24 - ABRAHÃO et al, 2004), Formol 10\% (F-12 e F-24 - SALMITO-VANDERLEY et al, 2002) e Paraformaldeído 4\% (P-12 e P-24 - SILVA et al., 2004). Cada ovário constituiu uma repetição.

Figura 1. Protocolo experimental utilizado em cada ovário para testar a eficiência de diferentes fixadores e tempos de fixação sobre a morfologia de folículos ovarianos pré-antrais.

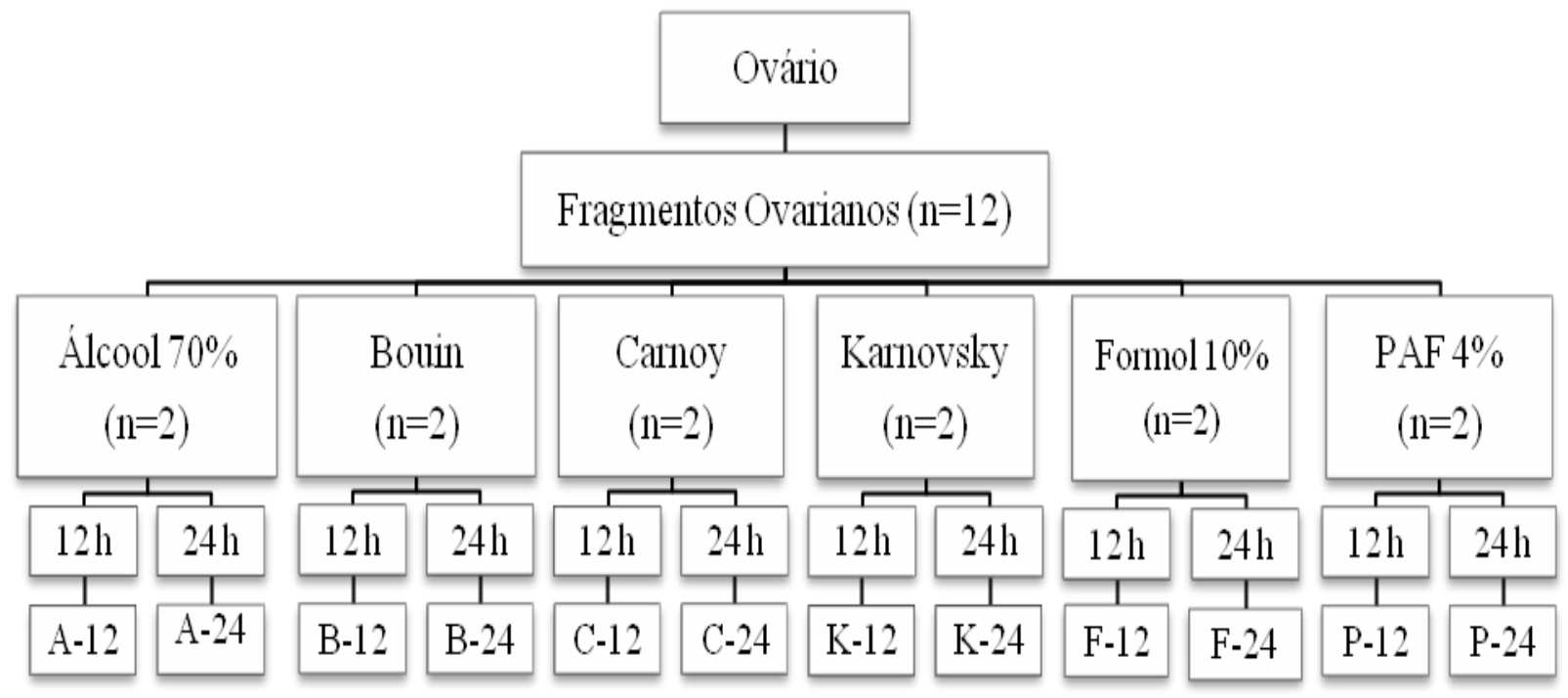

Fonte: Elaboração dos autores.

\section{Processamento histológico}

Após fixados por 12 ou $24 \mathrm{~h}$, os fragmentos foram acondicionados em álcool $70 \%$ por aproximadamente 24h. Para processamento histológico, os fragmentos foram desidratados em concentrações crescentes de álcool, diafanizados em xilol e incluídos em Paraplast Plus (Ted Pella, Inc - CA - USA). Posteriormente, foram realizados cortes seriados de $5 \mu \mathrm{m}$ em micrótomo rotativo (Leica ${ }^{\circledR}$, Wetzlar-Alemanha), seguidos de montagem em lâmina e coloração com ácido periódico de Schiff (PAS) e Hematoxilina. A leitura das lâminas foi realizada em microscópio óptico (Nikon®, Tokyo-Japão). Em cada tratamento foram avaliados 30 folículos por repetição, totalizando 150 folículos. De acordo com a morfologia os folículos pré-antrais foram classificados como degenerados quando apresentaram um ou mais dos seguintes aspectos: retração do citoplasma, núcleo picnótico, vacúolos citoplasmáticos, deslocamento das células da granulosa e rompimento da membrana basal (LUCCI et al., 2002).

\section{Análise estatística}

O delineamento experimental foi do tipo inteiramente casualizado com cinco repetições e 12 tratamentos. Os dados de viabilidade folicular foram submetidos à análise de variância utilizando modelos mistos lineares (mixed models; SAS, versão 9.1; SAS Institute Inc., Cary, NC, USA), sendo que a porcentagem de folículos viáveis em cada ovário consistiu uma repetição. As comparações múltiplas entre os grupos experimentais foram realizadas pelo LS means. O nível de significância considerado foi $\mathrm{P}<0,05$. 


\section{Resultados}

Foram avaliados 1800 folículos pré-antrais incluídos no tecido ovariano fixados em seis diferentes fixadores, durante 12 ou 24 horas. Tecidos fixados em Carnoy e Bouin por 12 e 24 horas apresentaram maior porcentagem $(\mathrm{P}<0,05)$ de folículos morfologicamente viáveis (88 e $83 \%$ para o fixador Carnoy vs. 79 e $73 \%$ para o Bouin, respectivamente) comparado aos demais fixadores. Entretanto, ambos fixadores não diferiram $(\mathrm{P}>0,05)$ entre si (Figura 2).

Figura 2. Porcentagem de folículos pré-antrais morfologicamente normais em tecidos ovarianos mantidos em seis fixadores diferentes por 12 ou 24 horas. Letras minúsculas indicam diferença a, b, c, d diferem entre si $(\mathrm{P}<0,05)$.

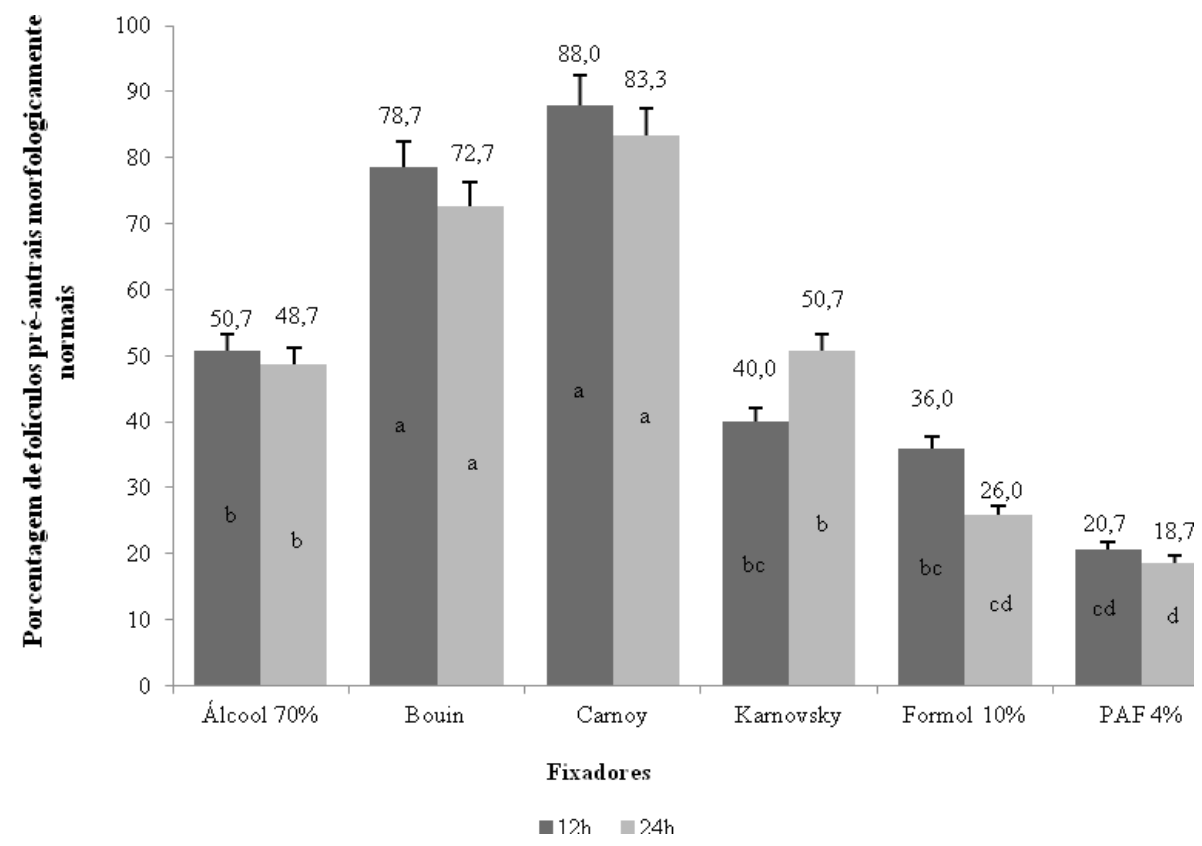

Fonte: Elaboração dos autores.

Tabela 1. Porcentagem de alterações morfológicas observadas em folículos ovarianos pré-antrais bovinos $(\mathrm{n}=150 /$ tratamento) mantidos em diferentes fixadores por 12 ou 24 horas. RC- retração citoplástica, VC- vacuolização do citoplasma, PC- picnose nuclear, DCG- deslocamento de células da granulosa, RMB- rompimento da membrana basal.

\begin{tabular}{|c|c|c|c|c|c|c|}
\hline \multirow{2}{*}{ Tratamentos } & \multicolumn{6}{|c|}{ Classificação das Alterações Morfológicas (\%) } \\
\hline & Total & $\mathrm{RC}$ & $\mathrm{VC}$ & $\mathrm{PC}$ & DCG & RMB \\
\hline Álcool 70\% $12 \mathrm{~h}$ & $49(74 / 150)$ & 59 & 14 & 28 & 47 & 1 \\
\hline Álcool 70\% 24 h & $51(77 / 150)$ & 40 & 10 & 36 & 47 & 1 \\
\hline Bouin $12 \mathrm{~h}$ & $21(32 / 150)$ & 28 & 22 & 56 & 34 & 3 \\
\hline Bouin $24 \mathrm{~h}$ & $27(41 / 150)$ & 32 & 22 & 39 & 37 & 5 \\
\hline Carnoy $12 \mathrm{~h}$ & $12(18 / 150)$ & 56 & 11 & 0 & 72 & 0 \\
\hline Carnoy $24 \mathrm{~h}$ & $17(25 / 150)$ & 56 & 20 & 16 & 72 & 0 \\
\hline Karnovsky $12 \mathrm{~h}$ & $60(90 / 150)$ & 43 & 12 & 19 & 60 & 6 \\
\hline Karnovsky 24 h & $49(74 / 150)$ & 50 & 11 & 27 & 54 & 8 \\
\hline Formol $10 \% 12 \mathrm{~h}$ & $64(96 / 150)$ & 53 & 8 & 22 & 60 & 6 \\
\hline Formol $10 \% 24 \mathrm{~h}$ & $74(111 / 150)$ & 57 & 7 & 16 & 73 & 8 \\
\hline Paraformaldeído $4 \% 12 \mathrm{~h}$ & $79(119 / 150)$ & 66 & 10 & 8 & 76 & 5 \\
\hline Paraformaldeído 4\% 24h & $81(122 / 150)$ & 63 & 14 & 9 & 83 & 7 \\
\hline
\end{tabular}

*RC- retração citoplástica, VC- vacuolização do citoplasma, PC- picnose nuclear, DCG- deslocamento de células da granulosa, RMB- rompimento da membrana basal. Fonte: Elaboração dos autores. 
A porcentagem de alterações morfológicas identificadas nos folículos degenerados em cada tratamento está apresentada na Tabela 1. As principais alterações encontradas foram retração citoplasmática e deslocamento de células da granulosa. Estes defeitos eram frequentemente observados em conjunto.

\section{Discussão}

No presente trabalho, apresentamos pela primeira vez uma comparação da eficiência de seis diferentes fixadores histológicos e dois tempos de fixação na preservação da morfologia de estruturas ovarianas de bovinos. O tipo de fixador utilizado pode preservar diferentemente detalhes morfológicos dos mais variados tipos de órgãos e células (BENNETT; WIRINCK; LEE, 1976; FERREIRA; CHIARINIGARCIA, 1992; SOARES et al., 2006). Embora diferentes fixadores sejam utilizados na rotina para a fixação do tecido ovariano, neste trabalho foi demonstrado que os fixadores Carnoy e Bouin apresentam melhores resultados em termos de manutenção da morfologia da estrutura ovariana em comparação aos demais fixadores testados. O melhor resultado de fixação é buscado principalmente em estudos que visem comparar a degeneração de folículos ovarianos pré-antrais (FOPA), uma vez que, os resultados tornam-se mais fidedignos quanto maior a eficiência do fixador.

A utilização da solução de Carnoy proporcionou a manutenção da estrutura ovariana, o que pode ser constatado pela alta porcentagem de FOPA que permaneceram morfologicamente normais após a fixação tecidual. Culling, Allisson e Barr (1985) descreveram que o Carnoy penetra rapidamente nos em diversos tecidos dando uma excelente fixação nuclear e preservação das estruturas. Tendo em vista, além da avaliação dos FOPA, também a pesquisa de patologias ovarianas por meio de histologia é interessante a validação de um fixador para este tipo de tecido.
Os fixadores coagulantes, como a solução de Bouin podem causar despolimerização das proteínas tissulares, fazendo com que as células e tecidos apresentem aspecto não homogêneo, o que dificulta a observação de detalhes citológicos (COLE; SYKES, 1974; LOCQUIN; LANGERON, 1983). Entretanto, cortes com aspecto histológico e coloração homogêneos, e de fácil avaliação dos detalhes foliculares foram observados nos tecidos ovarianos fixados com Carnoy e Bouin. Os folículos ovarianos degenerados fixados em solução de Bouin apresentaram alterações foliculares associadas à coloração do núcleo, como picnose nuclear, com 56 e 48\% das alterações observadas, 12 e $24 \mathrm{~h}$ pós-fixação. A picnose nuclear foi descrita como o primeiro sinal de atresia folicular (JORIO; MARIANA; LAHLOU-KASSI, 1991; WOOD; MONTALI; WILDT, 1997). Portanto, as alterações relacionadas à picnose nuclear do oócito bovino podem ser decorrentes da atresia ovariana e não do processo de fixação utilizado.

A retração tecidual foi outro fator relacionado ao tipo de fixador utilizado. Os folículos pré-antrais presentes no tecido ovariano fixado com formol $10 \%$ ou paraformaldeído $4 \%$ apresentaram grande número de defeitos relacionados com retração citoplasmática e deslocamento de células da granulosa, assim como retração tecidual total. Os aldeídos, como a formalina, por serem fixadores não coagulantes, fazem com que as proteínas celulares assumam aspecto de gel transparente. Este aspecto é resultante das ligações cruzadas formadas entre as moléculas do fixador e as macromoléculas dos tecidos. Dessa forma, após o processo de fixação, muitas moléculas de água permanecem ligadas aos tecidos e às macromoléculas tissulares às quais a água normalmente se encontra associadas, resultando em maior estabilização estrutural e consequentemente melhor preservação histológica (FOX et al., 1985; SESSO, 1998) o que, no entanto, não foi observado quando utilizamos formol 10\% ou paraformaldeído 4\% para fixação dos fragmentos ovarianos bovinos. Retração citoplasmática e deslocamento das células 
da granulosa também foram observados nos tecidos fixados no álcool 70\%. De acordo com Buesa (2008), fixadores alcoólicos provocam altos índices de retração tecidual, o que justificaria tais alterações.

\section{Conclusões}

Os fixadores Carnoy e Bouin são os mais indicados para a fixação do tecido ovariano, por período de 12 ou 24h, pois preservaram a morfologia dos folículos ovarianos pré-antrais.

\section{Agradecimentos}

M.M. Seneda é pesquisador bolsista de produtividade do CNPq e E.R. Andrade é bolsista DTI / CNPq.

\section{Referências}

ABRAHÃO, D. S.; TOLEDO-PIZA, A. R.; MARTINS, M. A.; SILVA NETO, J. C.; FERREIRA, E. C. J.; RAPADO, L. N.; HOSODA, T. M.; SILVA, R. C.; AZZUZ, A. C.; TANIWAKI, N. N.; PIRES, M. F. C. Estudo comparativo com diversos fixadores para aplicação em microscopia eletrônica de transmissão. Revista do Instituto Adolfo Lutz, São Paulo, v. 63, n. 2, p. 248-254, 2004.

ANDRADE, E. R.; SENEDA, M. M.; ALFIERI, A. A.; OLIVEIRA, J. A.; FIGUEIREDO, J. R.; TONIOLLI, R. Efeito de diferentes concentrações de ácido 3-indolacético na ativação e crescimento in vitro de folículos pré-antrais ovinos. Arquivo Brasileiro de Medicina Veterinária e Zootecnia, Belo Horizonte, v. 57, n. 3, p. 334-339, 2005.

BAUMGARTNER, W.; DETTINGER, H.; SCHMEER, N.; HOFFMEISTER, E. Evaluation of different fixatives and treatments for immunohistochemical demonstration of Coxiella burnetii in Paraffin-Embedded Tissues. Journal of Clinical Microbiology, Washington, v. 26, n. 10, p. 2044-2047, 1988.

BENNETT, H. S.; WIRINCK, A. D.; LEE, S. W. Science and art in preparing tissues embedded in plastic for light microscopy, with special reference to glycol methacrylate, glass knives and simple stains. Stain Technology, Baltimore, v. 51, n. 2, p. 71-97, 1976.
BUESA, R. J. Histology without formalin? Annals of Diagnostic Pathology, Philadelphia, n. 12, p. 387-396, 2008.

CHAVES, R. N.; MARTINS, F. S.; SARAIVA, M. V. A.; CELESTINO, J. J. H.; LOPES, C. A. P.; CORREIA, J. C.; LIMA VERDE, I. B.; MATOS, M. H. T.; BÁO, S. N.; NAME, K. P. O.; CAMPELLO, C. C.; SILVA, J. R. V.; FIGUEIREDO, J. R. Chilling ovarian fragments during transportation improves viability and growth of goat preantral follicles cultured in vitro. Reproduction Fertility and Development, East Melbourne, v. 20, n. 5, p. 640-647, 2008.

COLE, M. B.; SYKES, S. M. Glycol methacrylate in light microscopy: a routine method for embedding and sectioning animal tissue. Stain Technology, Baltimore, v. 49, n. 6, p. 387-400, 1974.

CULling, C. F. A.; ALlisSON, R. T.; BARR, W. T. Cellular pathology technique. 4. ed. London: Butterworth-Heinemann, 1985.

FERREIRA, R. M. A.; CHIARINI-GARCIA, H. Efeito da fixação e do meio de inclusão na preservação histológica do intestino da traíra Hoplias malabaricus (Bloch, 1794). Revista Brasileira de Ciências Morfológicas, São Paulo, v. 9, p. 32-37, 1992.

FIGUEIREDO, J. R.; CELESTINO, J. J. H.; RODRIGUES, A. P. R.; SILVA, J. R. V. Importância da biotécnica de MOIFOPA para o estudo da foliculogênese e produção in vitro de embriões em larga escala. Revista Brasileira de Reprodução Animal, Belo Horizonte, v. 31, n. 2, p. 143-152, 2007.

FOX, C. H.; JOHNSON, F. B.; WHITING, J.; ROLLER, P. P. Formaldehyde fixation. Journal of Histochemistry \& Cytochemistry, Baltimore, v. 33, n. 8, p. 845, 1985.

GILLESPIE, J. W.; BEST, C. J.; BICHSEL, V. E.; COLE, K. A.; GREENHUT, S. F.; HWITT, S. M.; AHRAM, M.; GATHRIGHT, Y. B.; MERINO, M. J.; STRAUSBERG, R. L.; EPSTEIN, J. I.; HAMILTON, S. R.; GANNOT, G.; BAIBAKOVA, G. V.; CALVERT, V. S; FLAIG, M. J.; CHUARQUI, R. F.; HERRING, J. C.; PFEIFER, D.; PETRICOIN, E. F.; LINEHAN, W. M.; DURAY, P. H.; BOVA, G. S.; EMMERT-BURCK, M. R. Evaluation of non-formoldeído in tissue fixation for molecular profiling studies. American Journal of Pathology, New York, v. 160, n. 2, p. 449-457, 2002.

JORIO, A.; MARIANA, J. C.; LAHLOU-KASSI, A. Development of the population of ovarian follicles during the prepubertal period in D'man and Timahdit sheep. Animal Reproduction Science, Amsterdam, v. 26, p. 239-250, 1991. 
JUNQUEIRA, L. C.; CARNEIRO, J. Histologia básica. 11. ed. Rio de Janeiro: Guanabara Koogan, 2008.

LOCQUIN, M.; LANGERON, M. Handbook of microscopy. London: Butterworths, 1983. 322 p.

LUCCI, C. M.; RUMPF, R.; FIGUEIREDO, J. R.; BÁO, S. N. Zebu (Bos indicus) ovarian preantral follicles: morphological characterization and development of an efficient isolation method. Theriogenology, Stoneham, v. 57, n. 5, p. 1467-1483, 2002.

MATOS, M. H. T.; ANDRADE, E. R.; LUCCI, C. M.; BAO, S. N.; SILVA, J. R. V.; SANTOS, R. R.; FERREIRA, M. A. L.; COSTA, S. H. F.; CELESTINO, J. J. H.; FIGUEIREDO, J. R. Morphological and ultrastructural analysis of sheep primordial follicles preserved in $0.9 \%$ saline solution and TCM 199. Theriogenology, Stoneham, v. 62, n. 1-2, p. 65-80, 2004.

SALMITO-VANDERLEY, C. S. B.; NEVES, M. M.; SANTANA, C. V.; MARQUES JUNIOR, A. P.; VASCONCELOS, A. C.; NUNES, J. E. S. Preservação do sistema genital de cabras para a avaliação em microscopia de luz. Arquivo Brasileiro de Medicina Veterinária e Zootecnia, Belo Horizonte, v. 54, n. 1, p. 100-102, 2002.
SAUMANDE, J. La Folliculogenese chez les ruminants. Recueil De Médecine Vétérinaire, Paris, v. 167, n. 34, p. 205-218, 1991.

SESSO, A. Fixação de sistemas biológicos. In: SOUZA, W. Técnicas básicas de microscopia eletrônica aplicada às ciências biológicas. Rio de Janeiro: Universidade Estadual Norte Fluminense, 1998. p. 1-17.

SILVA, J. R. V.; VAN DEN HURK, R.; COSTA, S. H. F.; ANDRADE, E. R., NUNES, A. P. A.; FERREIRA, F. V. A.; LÔBO, R. N. B.; FIGUEIREDO, J. R. Survival and growth of goat primordial follicles after in vitro culture of ovarian cortical slices in media containing coconut water. Animal Reproduction Science, Amsterdam, v. 81, n. 3-4, p. 273-286, 2004.

SOARES, J. M.; BELETTI, M. E; MACHADO, E. R.; SILVA, M. Histomorfometria de testículos de gatos (Felis domestica) utilizando-se três diferentes fixadores. Bioscience Journal, Uberlândia, v. 22, n. 1, p. 175-181, 2006.

WOOD, T. C.; MONTALI, R. J.; WILDT, D. E. Follicleoocyte atresia and temporal taphonomy in cold-stored domestic cat ovaries. Molecular Reproduction and Development, Malden, v. 46, n. 2, p. 190-200, 1997. 
